\title{
High Spin Cobalt Complexes Supported by a Trigonal Tris(Phosphin- imide) Ligand
}

\author{
Heui Beom Lee ${ }^{\dagger}$, Nicholas Ciolkowski ${ }^{\dagger}$, Charles Winslow ${ }^{\dagger}$, and Jonathan Rittle ${ }^{\dagger, *}$ \\ $\dagger$ Department of Chemistry, University of California Berkeley, Berkeley, California 94720, United States
}

\begin{abstract}
Terminal, $\pi$-basic moieties occupy a prominent position in the stabilization of unusual or reactive inorganic species. The electron-releasing, $\pi$-basic properties of phosphinimides (PN) have been employed to stabilize electrondeficient early transition metals and lanthanides. In principle, a ligand field comprised of terminal PN groups should enable access to high-valent states of late first row transition metals. Herein, we report a new class of multidentate phosphinimide ligands to logically explore this hypothesis. Access to such ligands is made possible by a new procedure for the electrophilic amination of rigid, sterically-encumbering, multidentate phosphines. Such frameworks facilitate terminal PN coordination to Cobalt as demonstrated by the synthesis of a trinuclear $\mathrm{Co}_{3}{ }_{3}$ complex and a homoleptic, three-coordinate $\mathrm{Co}^{\mathrm{III}}$ complex. Interestingly, the Co ${ }^{\text {III }}$ complex exhibits an exceedingly rare $S=2$ ground state. Combined XRD, magnetic susceptibility, and DFT studies highlight that terminally-bound PNs engage in strong $d \pi-p \pi$ interactions that present a weak ligand field appropriate to stabilize high-spin states of late transition metals.
\end{abstract}

\section{INTRODUCTION}

The unique features of anionic phosphinimides (PN) also known as imidophosphoranes - warrant further consideration in the stabilization of reactive inorganic fragments. ${ }^{1-2}$ The two canonical resonance structures of PNs (Fig 1A) highlight their ability to act as 1,3 , or 5 electron donors, attendant with the diversity of observed [PN-M] metal binding modes (Fig 1B). In the case of a terminally bound $\mathrm{PN}$ where the $\angle(\mathrm{P}-\mathrm{N}-\mathrm{M})$ angle approaches $180^{\circ}$, the PN moiety can serve as a 5 electron donor engaging in $\pi$-bonding with the metal center in two orthogonal planes, akin to imido ligands. ${ }^{3-4}$ The effectiveness of PNs as electron releasing substituents or donors has been exploited in the synthesis of phosphazene superbases and stable complexes of strongly oxidizing ions such as $\mathrm{Ce}(\mathrm{IV})$ and $\mathrm{Tb}(\mathrm{IV})$, respectively. ${ }^{5-7}$ As ligands, PNs also enable the generation of reactive, low-coordinate metal fragments via the incorporation of sterically-encumbering substituents on the phosphorus atom. This feature has been successfully used to generate a class of Ti-promoted olefin polymerization catalysts ${ }^{8-9}$ and $\mathrm{Ni}$ - or Co-promoted hydrogenation catalysts. ${ }^{10}$

High-valent states of late transition metal complexes are implicated as reactive intermediates in numerous synthetic and biological processes; isolation and characterization of such species is of great interest. ${ }^{11-12}$ In this context, the coordination of PNs to late transition metals as multielectron donors may be expected to stabilize high valent states of these metal ions. Excluding a small number of Ru and Os-PN complexes, ${ }^{13-16}$ very few mononuclear late transition metal-PN complexes have been reported (Fig 1B), often requiring the presence of sterically-demanding co-ligands to prevent oligomerization. 17-20 This situation can be partially explained by significant $d \pi-p \pi$ repulsive effects which are expected to impede the ability of
PN ligands to serve as 3- or 5-electron donors. This general tendency of late transition metal-PN complexes to form oligomers - even with the usage of sterically-encumbering $\mathrm{PN}$ ligands such as $t-\mathrm{Bu}_{3} \mathrm{PN}^{-}-$challenges synthetic efforts in this area. ${ }^{10,21}$

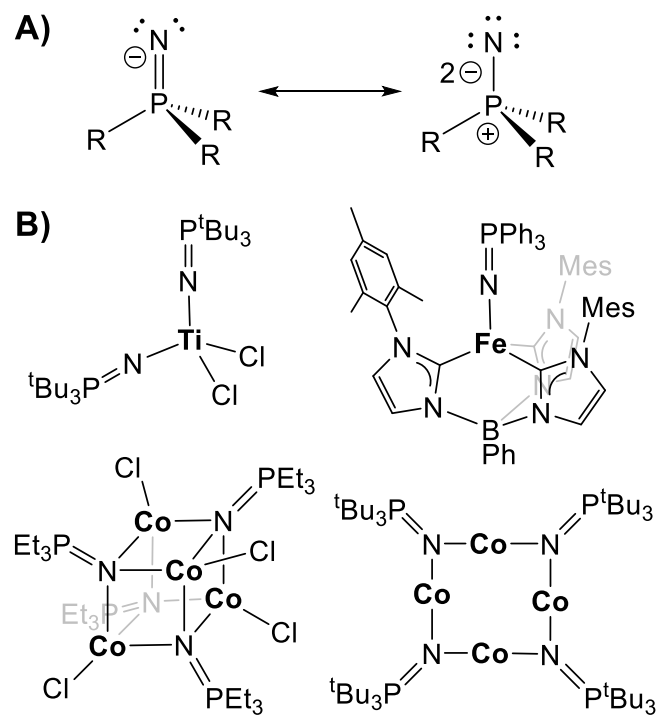

Figure 1. A) Canonical resonance structures of a phosphinimide (PN) functional group. B) Selection of metal-PN complexes, showing the diversity of metal binding modes and the propensity to make oligomeric structures with late first row transition metals. 
We hypothesized that the logical exploration of late transition metal-PN chemistry could be facilitated via the development of rigid, multidentate PN platforms. Specifically, ligands designed to enforce quasilinear P-N-M linkages could maximize $\pi$-donation to a bound metal ion and enable the synthesis of stable, high valent complexes with unusual electronic properties or reactivity profiles. Herein, we report a mild, high yielding, and general synthetic procedure for the synthesis of phosphinimines $\left(\mathrm{R}_{3} \mathrm{PNH}\right)$ from the corresponding phosphines. This protocol was instrumental in the synthesis of a chelating, tris-PN framework featuring a rigid triarylbenzene backbone that stabilizes both mono- and multinuclear transition metal complexes. A trinuclear $\mathrm{Co}_{3}{ }_{3}$ compound was found to react cleanly with $\mathrm{O}_{2}$ or $\mathrm{C}_{2} \mathrm{Cl}_{6}$ to afford an exceptionally-stable, high spin $(S=2)$ Co'll complex. Experimental and computational studies corroborate strong metal-ligand multiple bonding to the terminally-bound PN ligands which serve to stabilize this unusual spin state for $\mathrm{Co}^{\prime \prime \prime}$. Our results highlight the importance of ligand design in harnessing the $\pi$-basicity of PNs for the synthesis of complexes with unusual geometric and electronic structures, and provide the synthetic groundwork for the general investigation of PNs with late transition metals.

\section{RESULTS AND DISCUSSION}

We reasoned that terminal PN coordination to kinetically labile, late first row transition metals would be enforced via the incorporation of such ligands into a rigid, multidentate framework. To date, however, very few multidentate PN scaffolds have been prepared, and these suffer from pronounced flexibility and concomitant ambiguity in their metal coordination chemistry. ${ }^{22-26}$ Hence, a tris-PN pro-ligand $\left(\mathrm{H}_{3}{ }^{\mathrm{R}} \mathrm{L}\right)$ based on a rigid triarylbenzene backbone was designed (Scheme 1A). Using a similar backbone, tris-alkoxide, -siloxide, and -carboxylate variants have been described, ${ }^{27-29}$ and tris-phosphine precursors (Int1) bearing phenyl- or adamantyl substituents are readily prepared (Supporting Information) ${ }^{30-31}$ However, attempts to convert Int1 to $\mathrm{H}_{3}{ }^{\mathrm{R}} \mathrm{L}$ via traditional routes, including the usage of $\mathrm{Me}_{3} \mathrm{SiN}_{3}{ }^{6}$ or aminolysis of $\mathrm{R}_{3} \mathrm{PCl}_{2}$ derivatives $^{32}$ proved unsuccessful; even after prolonged
Scheme 1. (A) Synthesis of the tris-phosphinimine proligands $\mathrm{H}_{3}{ }^{\mathrm{R}} \mathrm{L}$ and (B) complexes $\mathbf{1}$ and $\mathbf{2}$ reported in this study.

A)
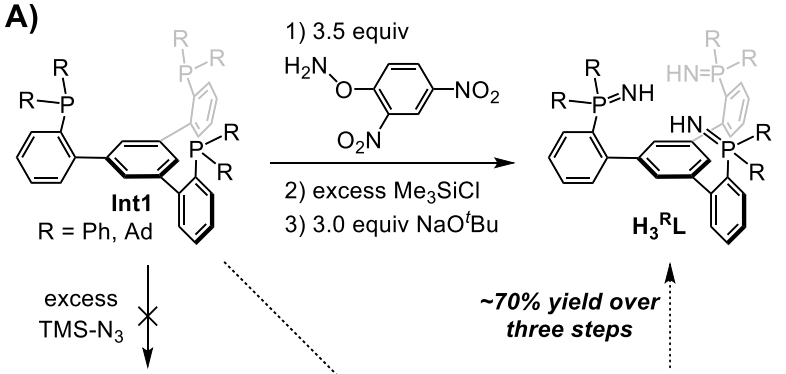

three steps
thield over
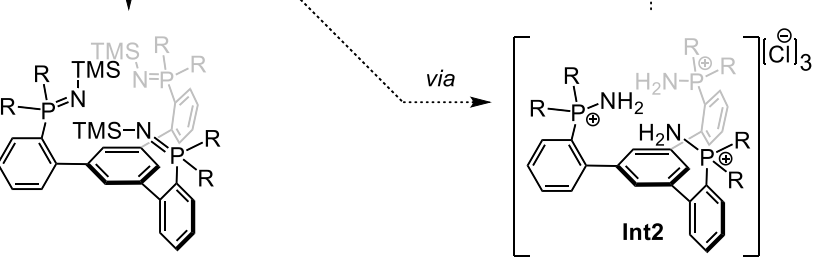

B)

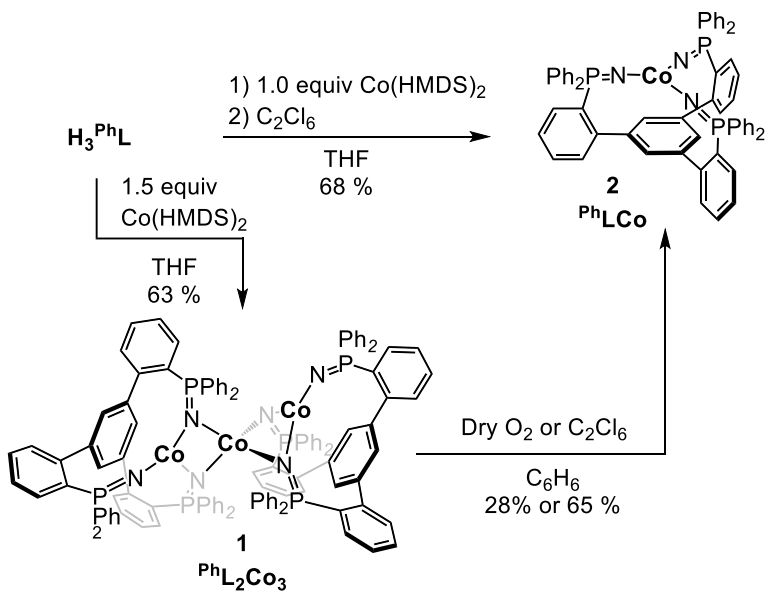

heating, incomplete conversion of PhInt1 was observed upon exposure to excess $\mathrm{Me}_{3} \mathrm{SiN}_{3}$ as monitored by ${ }^{31} \mathrm{P}$ NMR spectroscopy (Fig S18). We hypothesize that steric effects preclude incorporation of three bulky $\mathrm{Me}_{3} \mathrm{SiN}$ groups onto this constrained framework.

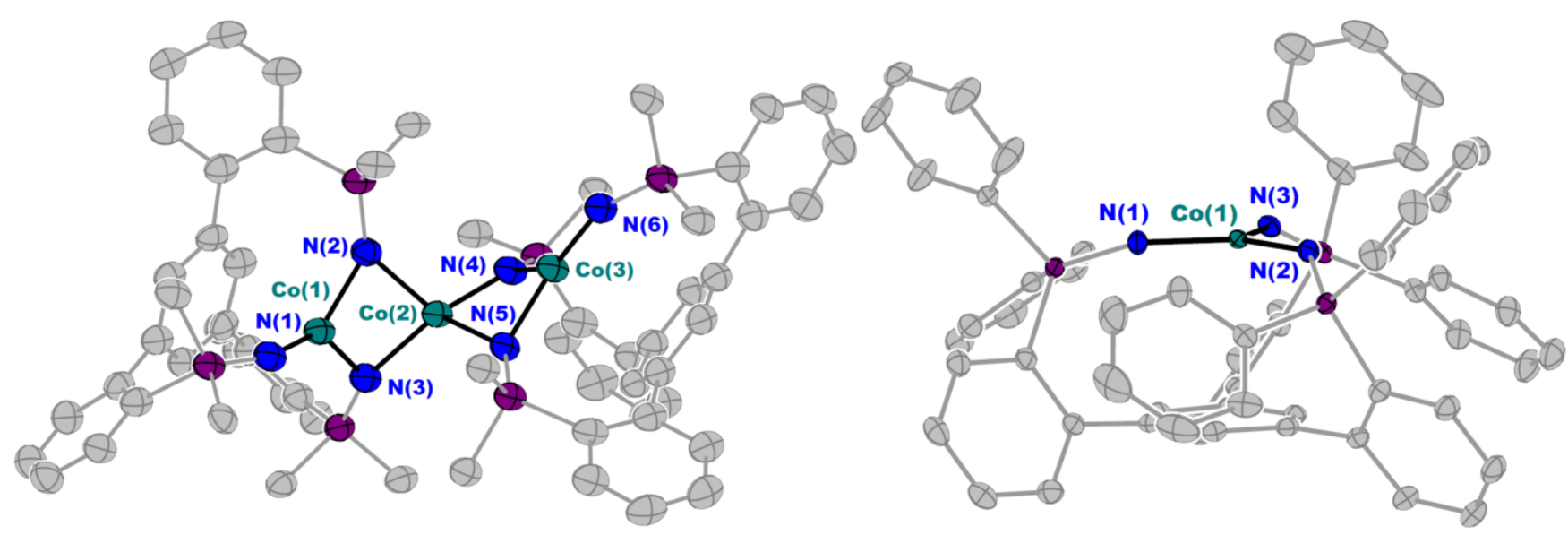

Figure 2. Crystal structure of 1 (left) and 2 (right) shown at $50 \%$ probability. Structure of 1 truncated for clarity. Selected bond distances (Å): 1: Co(1)-N(1) 1.862(4), Co(1)-N(2) 1.956(3), Co(1)-N(3) 1.992(3), Co(2)-N(2) 2.041(3), Co(2)-N(3) 2.029(3), Co(2)-N(4) 2.004(3), Co(2)-N(5) 2.042(3), Co(3)-N(4) 1.994(3), Co(3)-N(5) 1.959(3), Co(3)-N(6) 1.867(3); 2: Co(1)-N(1) $1.797(1), \mathrm{Co}(1)-\mathrm{N}(2) 1.826(1), \mathrm{Co}(1)-\mathrm{N}(3) 1.791(1)$. 
This observation underscores that the general application of PNs as ligands is limited by existing synthetic procedures for their preparation. We canvassed alternative reagents intended to effect the electrophilic amination of phosphines and identified O-(2,4-dinitrophenyl)-hydroxylamine as a non-hygroscopic, stable, and easily scalable reagent. ${ }^{33}$ The combination of this reagent with diverse mono- and diphosphines at room temperature results in quantitative conversion to the corresponding phospiniminium ions $\left(\mathrm{R}_{3} \mathrm{PNH}_{2}{ }^{+}\right)$as observed by ${ }^{31} \mathrm{P}-\mathrm{NMR}$ within 15 minutes, with isolated yields of $80-90 \%$ on gram scales (Supporting Information). Importantly, this reagent also quantitatively aminates all three phosphorus atoms of Int1 as monitored by ${ }^{31} \mathrm{P}$ NMR spectroscopy. To facilitate isolation, anion exchange by treatment with excess $\mathrm{Me}_{3} \mathrm{SiCl}$ afforded the iminium chloride Int2 as an insoluble solid. Further treatment of Int2 with three equivalents of $\mathrm{NaO}^{t} \mathrm{Bu}$ produced the desired tris-phosphinimines in $\sim 70 \%$ overall yield from Int1, providing efficient syntheses of the $\mathrm{H}_{3}{ }^{\mathrm{R}} \mathrm{L}$ pro-ligands.

Simultaneous metalation and conversion of the phenyldecorated pro-ligand $\left(\mathrm{H}_{3}{ }^{\mathrm{Ph}} \mathbf{L}\right)$ to its triply anionic, tris-PN form occurred upon treatment with a Coll amide synthon. Treatment of $\mathrm{H}_{3}{ }^{\mathrm{Ph}} \mathrm{L}$ with 1.5 equivalents of $\mathrm{Co}\left[\mathrm{N}\left(\mathrm{SiMe}_{3}\right)_{2}\right]_{2}$ (THF) cleanly furnishes the trinuclear complex 1 in $63 \%$ yield. The KBr-IR spectrum of 1 lacks features ascribable to $v(\mathrm{~N}-\mathrm{H})$ vibrations (Fig S27) and the $\mathrm{X}$ ray crystal structure corroborates a $\left[{ }^{\mathrm{Ph}} \mathrm{L}\right]_{2} \mathrm{Co}_{3}$ formulation (Fig 2, Scheme 1B). The structure can be interpreted as
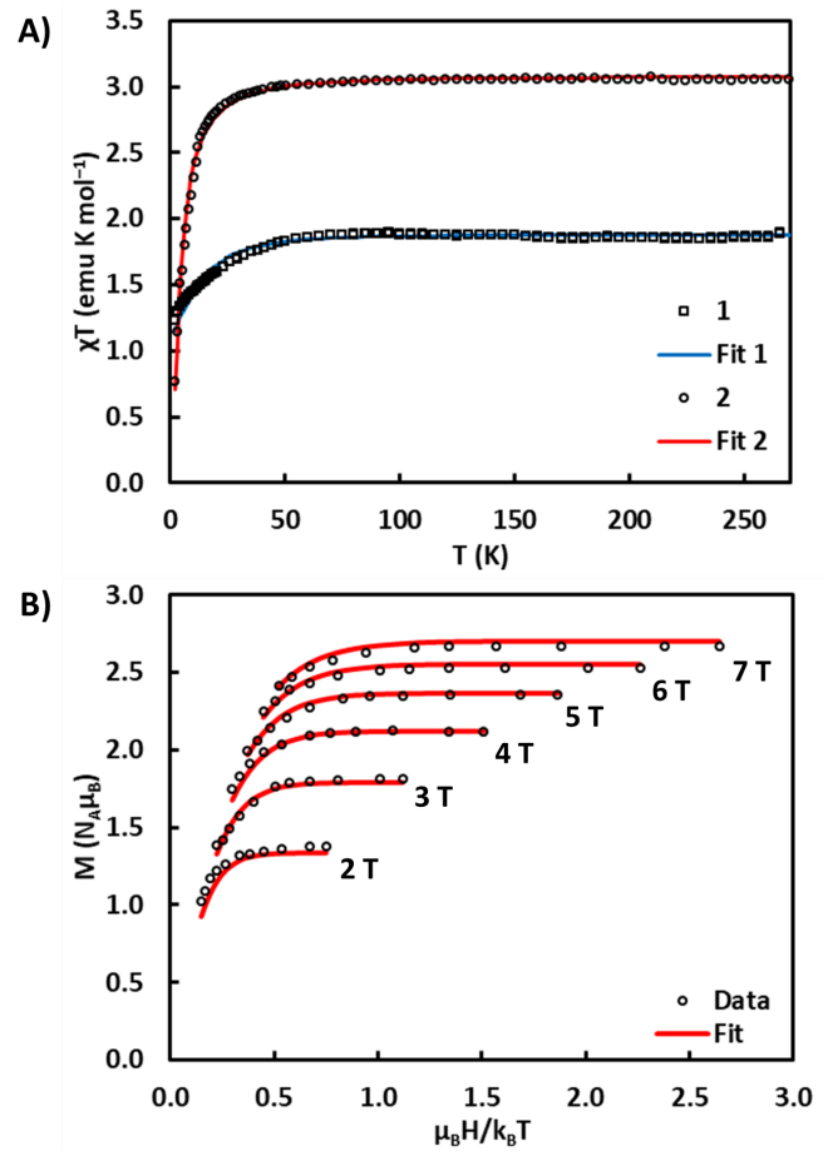

Figure 3. (A) $\chi T$ vs $T$ plot of the magnetic susceptibility data of $\mathbf{1}$ and $\mathbf{2}$ and corresponding fit. (B) Reduced magnetization plot of 2. See text for fit parameters. two $(\mathrm{PN})_{3} \mathrm{Co}^{\prime \prime}$ fragments (i.e. $\left.{ }^{\mathrm{Ph}} \mathrm{LCo}^{\prime \prime}\right]^{-}$) linked by a central, pseudo-tetrahedral $\mathrm{Co}^{\text {II }}$ ion. The geometry of the flanking Co centers is nearly trigonal planar, with the sum of the respective $\angle(\mathrm{N}-\mathrm{Co}-\mathrm{N})$ angles approaching $360^{\circ}\left(358.1^{\circ}\right.$ and $\left.358.6^{\circ}\right)$. The terminal Co-N distances are observed at 1.862(4) and 1.867(3) $\AA$; bridging Co-N distances are longer, in the range 1.96 2.04 $\AA$, consistent with distances reported in oligomeric Co-PN complexes. ${ }^{21,34}$ The structure of compound 1 recapitulates the strong tendency of late transition metals to bind PN ligands via bridging modes (Fig 1B). In principle, increasing the steric encumbrance of the flanking phosphorus substituents via usage of the $\mathrm{H}_{3}{ }^{\mathrm{Ad}} \mathbf{L}$ ligand variant may enable the synthesis of mononuclear Co" derivatives and efforts on this front are ongoing.

Magnetic susceptibility studies performed on 1 reveal a strongly antiferromagnetically coupled spin system. A near ideal Curie behavior was observed in the range of $50-270 \mathrm{~K}$ with a $\chi^{\mathrm{T}}$ value of $1.90 \mathrm{emu} \mathrm{K} \mathrm{mol}^{-1}$ (Fig 3A), consistent with the expected spin-only value of $1.875 \mathrm{emu}$ $\mathrm{K} \mathrm{mol}^{-1}(g=2)$ for an $S=3 / 2$ spin state. Employing the Hamiltonian $H=-2 J\left(S_{1} S_{2}+S_{2} S_{3}\right)$, the data was fit with $g=$ $2.00, J=-150 \pm 20 \mathrm{~cm}^{-1}$ for $\mathrm{T}>50 \mathrm{~K}$. The subscripts in the Hamiltonian follow the numbering scheme for the Co centers in the crystal structure of $\mathbf{1}$ (Fig 2). Due to the small curvature of the $\chi T$ vs T curve, a relatively large variance of $J$ is reported, and the fitted value should be taken as an estimate. However, the small temperature dependence of $\chi \mathrm{T}$ above $50 \mathrm{~K}$ suggests that the energy separation between the $S=3 / 2$ ground state and the $S=1 / 2$ and $S=5 / 2$ excited states are on the order of several hundred wavenumbers. Below $50 \mathrm{~K}, \chi^{\top}$ decreases with temperature, and a zero-field splitting parameter of $|D|=6.7 \mathrm{~cm}^{-1}$ was used to fit the data. The thermally well-isolated $S=$ $3 / 2$ ground state of 1 is consistent with strong pair-wise antiferromagnetic exchange coupling between three high spin $S=3 / 2$ Co"l centers.

Few magnetometry studies have been reported on multinuclear PN-bridged systems. On one hand, negligible exchange coupling was found for a dimeric Dy ${ }^{\prime \prime \prime}{ }_{2}$ complex bridged by two PN ligands. ${ }^{35}$ In contrast, PN-bridged, homovalent $\mathrm{Ni}_{4}{ }_{4}$ and $\mathrm{Co}_{4}{ }_{4}$ complexes ${ }^{36-37}$ exhibit well-isolated spin states attributed to the presence of direct metalmetal orbital overlap inferred by short $\mathrm{Ni}-\mathrm{Ni}$ and $\mathrm{Co}-\mathrm{Co}$ distances of $\sim 2.4 \AA$. Such direct overlap may be operative in 1 despite substantially longer Co-Co distances $(2.67$ and $2.70 \AA$ ). A cuboidal [Fe"Cl(NPEt 3$)]_{4}$ complex, featuring four $\mu_{3}-\mathrm{PN}$ ligands and Fe-Fe distances of $\sim 2.73 \AA$,
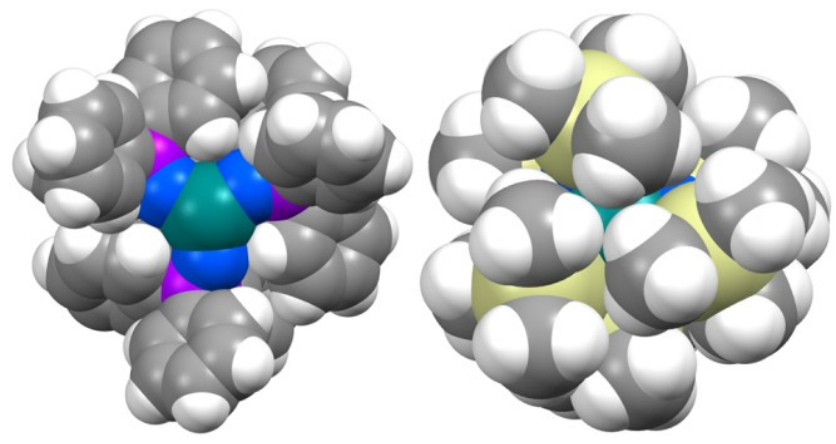

Figure 4. Spacefilling models of the crystal structure of 2 (left) and $\mathrm{Co}\left[\mathrm{N}\left(\mathrm{SiMe}_{3}\right)_{3}\right]_{3}$ (right), viewed normal to the plane defined by the three nitrogen atoms. 
displayed Curie-Weiss behavior in the temperature range of $30 \sim 150 \mathrm{~K}^{38}$ Taken together, these reports and the magnetometry data for 1 corroborate that bridging PN ligands can mediate strong antiferromagnetic exchange interactions with later transition metals.

We explored the oxidative chemistry of complex 1 in anticipation that higher valent states of cobalt may be stabi-

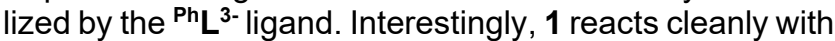
dry $\mathrm{O}_{2}$ in $\mathrm{C}_{6} \mathrm{D}_{6}$ over 24 hours to give a new paramagnetic species (compound 2). The ${ }^{1} \mathrm{H}-\mathrm{NMR}$ spectrum of 2 (Fig S25) exhibits six overlapping, paramagnetically-shifted resonances, consistent with a three-fold symmetric compound. Solution-phase magnetic measurements indicate that this species exhibits an $S=2\left(4.94 \mu_{\mathrm{B}}\right)$ spin state at room temperature. ${ }^{39}$ The X-ray crystal structure of 2 reveals a mononuclear $\left[{ }^{\mathrm{Ph}} \mathrm{L}\right] \mathrm{Co}$ compound featuring a formally-trivalent cobalt center bound to three terminal PN moieties (Fig 2). Apparently, the addition of $\mathrm{O}_{2}$ to 1 results in its chemical oxidation to afford 2 and an insoluble, uncharacterized $\mathrm{Co}_{x} \mathrm{O}_{y}$ byproduct. The conversion of $\mathbf{1}$ to $\mathbf{2}$ is also efficiently mediated by hexachloroethane $(65 \%$ yield), and compound $\mathbf{2}$ is most conveniently prepared in one pot via treatment of $\mathrm{H}_{3}{ }^{\mathrm{Ph}} \mathrm{L}$ with 1 equivalent of $\mathrm{Co}\left[\mathrm{N}\left(\mathrm{SiMe}_{3}\right)_{2}\right]_{2}$ (THF) followed by oxidation with 1 equivalent of $\mathrm{C}_{2} \mathrm{Cl}_{6}$ (68\% yield).

The high spin-state and the three-coordinate Co geometry of $\mathbf{2}$ is quite unusual, as the vast majority of molecular Co ${ }^{\text {III }}$ compounds are diamagnetic and five- or six-coordinate. $\mathrm{Co}\left[\mathrm{N}\left(\mathrm{SiMe}_{3}\right)_{2}\right]_{3}$ represents the only other example of a structurally characterized $S=2 \mathrm{Co}^{\text {III }}$ complex and its stability could be ascribed to the bulky amide ligands that occlude the coordination of additional ligands. ${ }^{40}$ In contrast, the space-filling representation of 2 (Fig 4) emphasizes a completely exposed Co center from the axial direction. Yet, compound $\mathbf{2}$ does not detectably coordinate
Lewis bases, such as DMAP, (Fig S26) and is crystallized from THF. Interestingly, the average Co-N distances in 2 $(1.805(1) \AA)$ are much shorter than those of $\mathrm{Co}\left[\mathrm{N}\left(\mathrm{SiMe}_{3}\right)_{2}\right]_{3}(1.870(3) \AA)$, consistent with substantial Co-N multiple bonding character in 2 . We speculate that strong, multiplanar Co-N $\pi$-bonding interactions serve to stabilize 2 and is corroborated by Density Functional Theory (DFT) studies (vide infra).

Magnetic susceptibility measurements of 2 support an isolated $S=2$ spin ground state. For polycrystalline 2 , an ideal Curie behavior was observed in the range of 50-300 $\mathrm{K}$ with a $\chi \mathrm{T}^{\mathrm{T}}$ value of 3.06 emu $\mathrm{K} \mathrm{mol}^{-1}$ (Fig $3 \mathrm{~A}$ ), consistent with the expected spin-only value of 3.0 emu $\mathrm{K} \mathrm{mol}^{-1}(\mathrm{~g}=$ 2 ) for an $S=2$ spin state. Below $50 \mathrm{~K}, \chi^{\top}$ decreases monotonically with temperature, indicating either the presence of significant zero-field splitting or spin crossover to $S=0$ or 1 . However, the reduced magnetization data ranging from 2-7 T and 1.8-9 K (Fig 3B) and the whole temperature range of the $\chi^{\top}$ data can be fit with a single set of parameters, suggesting that $\mathbf{2}$ does not detectably populate alternative spin states. The fit parameters are: $S=2$, $g=2.02, D=+11 \mathrm{~cm}^{-1}$. While intermediate $(S=1)$ and high spin $(S=2) C^{\prime \prime l}$ has been observed in oxide materials, ${ }^{41-43}$ molecular examples of either spin state remain rare. $\mathrm{K}_{3} \mathrm{CoF}_{6}$ has a moment of $5.60 \mu_{\mathrm{B}}$ at room temperature, consistent with the expected moment of $5.48 \mu_{\mathrm{B}}$ from an octahedral $\left[\mathrm{Co}^{11 \mathrm{II}} \mathrm{F}_{6}\right]^{3-}$ ion $(S=2, L=2){ }^{44}$ $\mathrm{Co}\left[\mathrm{N}\left(\mathrm{SiMe}_{3}\right)_{2}\right]_{3}$ has a moment of $4.73 \mu_{\mathrm{B}}$ at room temperature, consistent with the expected spin-only moment of $4.90 \mu_{\mathrm{B}}$ for $S=2 .{ }^{40}$ The relatively large, positive value of $D$ can be ascribed to the trigonal planar geometry in $2 .^{45}$ Consistent with a large positive $D$, no EPR transitions were observed at X-band in both perpendicular and parallel mode; EPR transitions may be observable at much higher frequencies but such studies were not pursued.

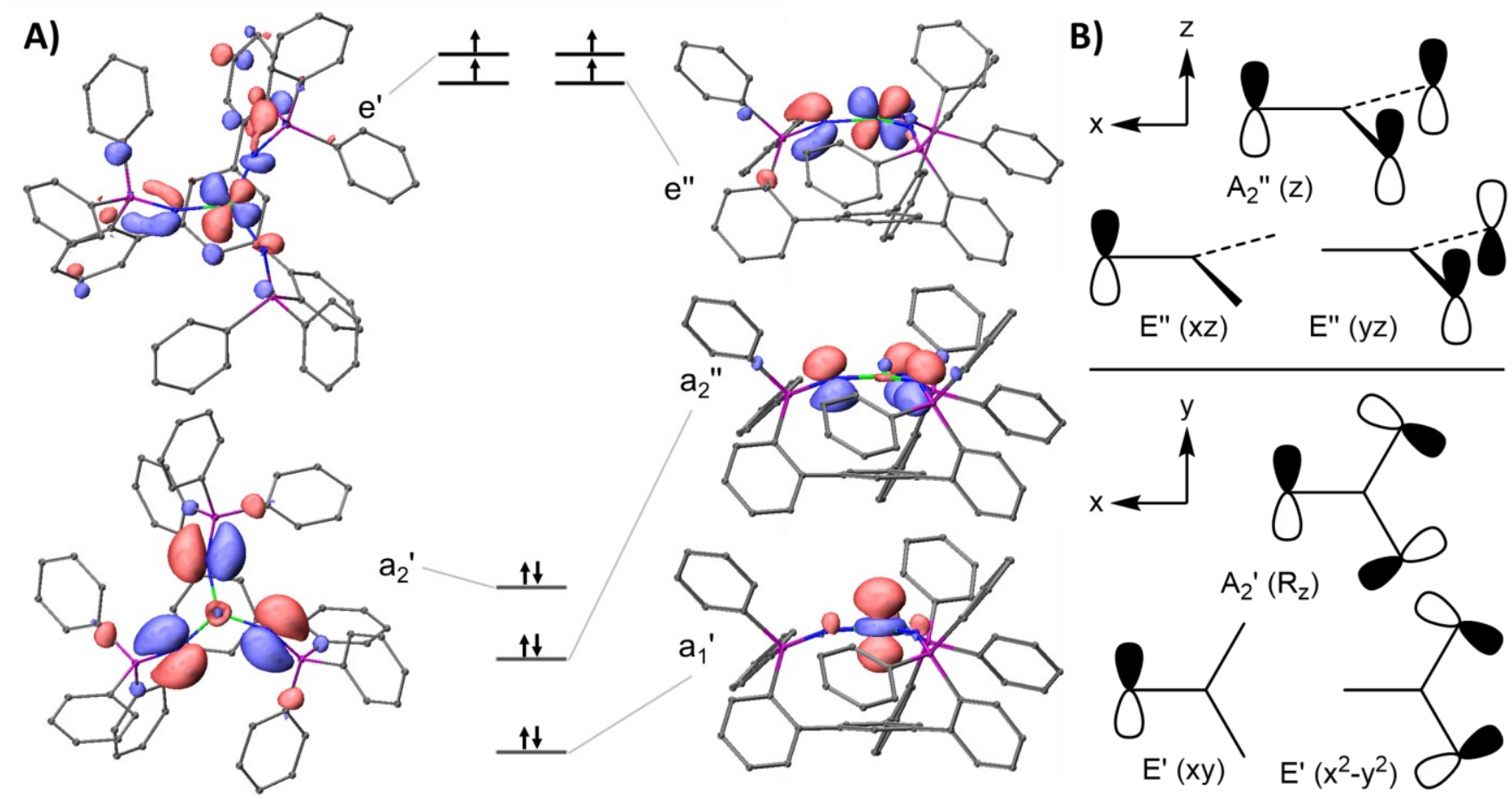

Figure 5. (A) Calculated, qualitative frontier orbital energy diagram for 2. Selected singly occupied orbitals rendered at 0.05 e/au ${ }^{3}$. Symmetry labels in an ideal $D_{3 \mathrm{~h}}$ symmetry. For a plot showing $\alpha$ and $\beta$ orbital energies separately, see Figure S34. (B) Symmetry adapted linear combinations for the PN HOMOs in an ideal $D_{3 \mathrm{~h}}$ symmetry. $\mathrm{P}$ atoms omitted for clarity. 
Electrochemical studies of $\mathbf{2}$ were pursued to understand the extent to which terminal PN ligation stabilizes the Co $\mathrm{Co}^{\text {III }}$ oxidation state. The cyclic voltammogram (CV) of 2 in a THF electrolyte features an irreversible reduction at $E=-1.67 \mathrm{~V}$ vs. $\mathrm{Fc} / \mathrm{Fc}^{+}$, which can be assigned to the reduction to Co" (Fig S32). An irreversible oxidation event at $\sim 0 \mathrm{~V}$ is also observed and can be attributed to a $\mathrm{Co}^{\prime \prime \prime} / \mathrm{Co}^{\mathrm{IV}}$ redox couple or oxidation of the ligand framework. The electrochemical data of 2 can be compared to [Co $\left.{ }^{\text {III }}(\mathrm{TAML})\right]^{-}$, a Co ${ }^{\text {III }}$ complex supported by a tetra-anionic, macrocyclic amide ligand. ${ }^{46}$ For $\left[\mathrm{Co}^{\text {III }}(\mathrm{TAML})\right]^{-}$, the $\mathrm{Co} / \mathrm{Co}^{\prime \prime l}$ couple was observed at $-1.18 \mathrm{~V}$ vs. $\mathrm{Fc}^{\prime} / \mathrm{Fc}^{+}$in a DCM electrolyte. The electrochemical data for 2 shows an enhanced stabilization $(\sim 0.5 \mathrm{~V})$ of the Co ${ }^{\text {III }}$ oxidation state as compared to anionic [Co"IIIAML) ${ }^{-}$. Accordingly, these data corroborate a hypothesis that terminally bound PN moieties quench the expected electrophilicity of $\mathrm{Co}^{\text {III }}$, and may explain the hesitancy of $\mathbf{2}$ to react with Lewis bases.

To better understand the unique Co-PN bonding situation in 2, DFT studies were performed. The calculated energies of the frontier orbitals are shown in Fig 5A. Consistent with the trigonal planar symmetry surrounding the Co center in $\mathbf{2}$ and the magnetometry data, the orbitals of $d$ parentage split into a 2-2-1 pattern with two sets of doubly degenerate orbitals; the orbital of $d_{z}^{2}$ parentage is lowest in energy and doubly occupied. The remaining four singly occupied orbitals each exhibit substantial Co- $\mathrm{N} \pi^{*}$ character (Fig 5A). This can be understood by considering an idealized $D_{3 h}$ arrangement of three terminal PN ligands (Fig 5B). In addition to the three $\mathrm{N}$-based symmetryadapted linear combinations (SALCs) that provide $\sigma$ bonding interactions with the central Co center, six $\mathrm{N}$ based SALCs are available for additional $\pi$-bonding interactions. The $A_{2}{ }^{\prime \prime}$ and $A_{2}{ }^{\prime}$ combinations do not possess the appropriate symmetry to overlap with $d$ orbitals, but the remaining four SALCs can interact with each orbital comprising the two degenerate e sets (i.e. $d_{x z}, d_{y z}, d_{x y}$ and $d_{x}{ }^{2}$ $\left.y^{2}\right)$. This bonding situation contrasts that found for related multidentate amide-based ligand architectures, such as $(\text { TAML })^{4-}$ or $(\text { TREN })^{3-}$ derivatives, which are limited to $\pi$ donation in a single orientation, either in the $x z / y z$ plane or the xy plane, respectively. Trigonal PN coordination results in the simultaneous destabilization of all $\pi$-symmetric $d$ orbitals, and the stabilization of high-spin ground states is further evident upon considering the small energy separation $(0.2 \mathrm{eV})$ between the Co- $\mathrm{N}$ antibonding $e^{\prime}$ and $e^{\prime \prime}$ sets in 2 (Fig 5A). To further illustrate this point at an energetic level, DFT studies employing identical levels of theory were performed on $\mathrm{Co}\left[\mathrm{N}\left(\mathrm{SiMe}_{3}\right)_{2}\right]_{3}$ (Fig S33). The separation between the corresponding $e^{\prime}$ and $e^{\prime \prime}$ sets in $\mathrm{Co}\left[\mathrm{N}\left(\mathrm{SiMe}_{3}\right)_{2}\right]_{3}$ is noticeably larger $(0.4 \mathrm{eV})$, likely because there is only one available set of $N$-based $\pi$-bonding SALCs that overlap efficiently with the $d$ orbitals (Fig S35). Finally, the UV-vis spectra of $\mathrm{Co}\left[\mathrm{N}\left(\mathrm{SiMe}_{3}\right)_{2}\right]_{3}$ and 2 suggest that the PNs contribute to a larger ligand field than amides, as indicated by the higher energy charge transfer transitions in 2 (Fig S30 and S38); a more detailed discussion can be found in the supporting information.

\section{CONCLUSIONS}

In summary, a methodology for the synthesis of phosphinimines from wide-ranging phosphine precursors has been developed to facilitate exploration of new avenues in molecular phosphinimide chemistry. Here, we applied this protocol to prepare rigid, multidendate, tris-phosphinimine scaffolds that serve to stabilize unique mono- and multinuclear cobalt complexes, including the stabilization of a rare, high-spin Co ${ }^{\text {III }}$ complex. Structural, computational and magnetometry data support the notion that a trigonally disposed tris(phosphinimide) ligand field is simultaneously weak field and strongly electron releasing. We envision that these new ligand frameworks will serve to stabilize unusual oxidation- and spin states of other late, first row transition metal ions, and efforts on this front are ongoing.

\section{EXPERIMENTAL SECTION}

General methods and instrumentation. All reactions were performed at room temperature in an $\mathrm{N}_{2}$-filled glovebox or by using standard Schlenk techniques unless otherwise specified. Glassware was oven dried at $150{ }^{\circ} \mathrm{C}$ for at least $2 \mathrm{~h}$ prior to use, and allowed to cool under vacuum. All reagents were used as received unless otherwise stated. Anhydrous pentane, $\mathrm{CH}_{3} \mathrm{CN}$, diethyl ether, benzene, toluene and THF were purified by sparging with nitrogen for 30 minutes and then passing under nitrogen pressure through a column of activated A2 alumina. NMR solvents were purchased from Cambridge Isotope Laboratories. $\mathrm{C}_{6} \mathrm{D}_{6}$ was degassed by three freeze-pump-thaw cycles and dried over freshly activated $3 \AA$ molecular sieves for 24 hours prior to use. Paramagnetic ${ }^{1} \mathrm{H}$ NMR spectra were recorded on Bruker $400 \mathrm{MHz}$ or $600 \mathrm{MHz}$ instruments, with shifts reported relative to the residual solvent peak. Elemental analyses were performed at the University of California Berkeley. FTIR measurements were carried out on a Thermo Scientific Nicolet iS20 spectrometer. UV-vis measurements were carried out on a Hewlett Packard 8453 UV-Visible spectrophotometer. Electrochemical measurements were carried out using a BioLogic SP50 potentiostat. Magnetic susceptibility measurements were carried on a Quantum Design MPMS $\mathrm{XL}$ instrument running MPMS Multivu software. Powdered, crystalline samples were placed inside a custommade quartz tube, vacuum sealed, and suspended in a plastic straw. Fitting simulations were performed using $\mathrm{PHI}^{47}$ The following compounds were synthesized according to published procedure: $\mathrm{Co}\left[\mathrm{N}\left(\mathrm{SiMe}_{3}\right)_{2}\right]_{2}(\mathrm{THF}),{ }^{48}$ 1,3,5-tris(2-bromophenyl)-benzene ${ }^{49} \quad$ O-(2,4-dinitrophenyl)-hydroxylamine, ${ }^{33} \mathrm{di}\left(1\right.$-adamantyl)phosphine. ${ }^{50}$

Detailed information on the synthesis of Int1, NMR spectra, X-ray diffraction, and DFT calculations are provided in the Supporting Information.

Caution! The electrophilic aminating agent O-(2,4-dinitrophenyl)-hydroxylamine and its derivatives are potentially energetic compounds in pure form. Preparations of this reagent as described ${ }^{33}$ typically afford material that is $\sim 95 \%$ pure, can be handled in air, and stored at $-35^{\circ} \mathrm{C}$ for months without noticeable decomposition. Representative samples do not exhibit characteristics of a thermallyinduced explosive (Fig S1) and are resistant to compression shock and sparks. Owing to the explosive nature of pure 2,4-dinitrophenol in air, all necessary safety precautions should be undertaken when handling the 2,4-dinitrophenolate intermediates derived from the amination procedures. Specifically, heating of reaction mixtures should 
be avoided, all manipulations should be performed under an inert atmosphere, and reaction byproducts should be diluted with water prior to disposal.

Synthesis of $\mathrm{H}_{3}{ }^{\mathrm{Ph}} \mathrm{L}$. All manipulations were performed inside a glovebox. A $500 \mathrm{~mL}$ round-bottom flask equipped with a stir bar was charged with PhInt1 $(27 \mathrm{~g}, 31 \mathrm{mmol}, 1$ equiv) and $\mathrm{MeCN}(300 \mathrm{~mL})$. The flask was cooled in a liquid nitrogen-chilled cold well. Solid O-(2,4-dinitrophenyl)-hydroxylamine ( $22 \mathrm{~g}, 110 \mathrm{mmol}, 3.5$ equiv) was added in small portions with stirring. The mixture was allowed to warm up to room temperature, resulting in the formation of a deep red solution with a yellow precipitate. The ${ }^{31} \mathrm{P}$ NMR of this red solution shows a single peak at $35 \mathrm{ppm}$, which corresponds to the chemical shift of the $\left[\mathrm{Ph}_{3} \mathrm{PNH}_{2}\right]^{+}$moiety (Figure S14). After stirring for 3 hours at room temperature, the flask was cooled again in a liquid nitrogen-chilled cold well. Neat $\mathrm{Me}_{3} \mathrm{SiCl}(35 \mathrm{~g}, 322 \mathrm{mmol}$, 10 equiv) that had been previously purified by short-path vacuum distillation was added in small portions with stirring. The mixture was allowed to warm up to room temperature, resulting in the formation of a yellow suspension. The mixture was further stirred at room temperature for 24 hours before the yellow precipitate was collected in a fritted funnel. The yellow solid was repeatedly washed with $\mathrm{MeCN}$ until the filtrate was colorless, subsequently washed with $\mathrm{Et}_{2} \mathrm{O}$, and thoroughly dried under reduced pressure. This insoluble yellow solid is assigned to the tris-phosphiniminium intermediate ${ }^{\mathrm{Ph}}$ Int2 with chloride counter-anions and was used without further purification. A $500 \mathrm{~mL}$ round-bottom flask equipped with a stir bar was charged with PhInt2 (25 g, 25 mmol, 1 equiv) and THF $(300 \mathrm{~mL})$. The flask was cooled in a liquid nitrogen-chilled cold well. Solid $\mathrm{NaO}^{t} \mathrm{Bu}$ (7.14 g, $74 \mathrm{mmol}, 3$ equiv) was added in small portions with stirring. The mixture was allowed to warm up to room temperature, resulting in the formation of a slightly beige solution over a brown precipitate. The mixture was further stirred at room temperature for 18 hours before being filtered through a pad of Celite. All volatiles were thoroughly removed from the filtrate. The beige residue was treated with $\mathrm{Et}_{2} \mathrm{O}$ and stirred until a fine suspension was obtained. The solid was collected in a fritted funnel, and washed with $\mathrm{Et}_{2} \mathrm{O}$ repeatedly. The collected solid was washed with small amounts of cold THF to remove colored impurities. The white solid was washed with pentane and dried under reduced pressure. $\mathrm{H}_{3}{ }^{\mathrm{Ph}} \mathrm{L}$ was obtained as a white solid $(20 \mathrm{~g}, 70 \%$ from PhInt1). ${ }^{1} \mathrm{H}$ NMR $\left(400 \mathrm{MHz}, \mathrm{CDCl}_{3}\right): 7.66-7.53$ (dd, $J=$ $11.9,7.2 \mathrm{~Hz}, 12 \mathrm{H}), 7.42-7.28(\mathrm{~m}, 21 \mathrm{H}), 7.19(\mathrm{t}, J=7.7$ $\mathrm{Hz}, 3 \mathrm{H}), 7.05-6.93(\mathrm{~m}, 6 \mathrm{H}), 6.60$ (s, 3H), 1.50 (bs, 3H, $\mathrm{NH}) \mathrm{ppm} .{ }^{31} \mathrm{P}$ NMR (162 MHz, $\left.\mathrm{CDCl}_{3}\right): 20.6 \mathrm{ppm} .{ }^{13} \mathrm{C}$ NMR (151 MHz, CDCl 3 ): 147.04, 146.68, 145.63, 145.43, $139.69,139.41,138.99,138.52,135.25,134.58,133.98$, $133.69,133.50,133.01,132.95,132.60,132.40,132.34$, $131.89,131.75,131.27,130.90,129.95,128.29,128.21$, 126.49, 126.26 ppm. HRMS (ESI): calcd. for $\mathrm{C}_{60} \mathrm{H}_{50} \mathrm{~N}_{3} \mathrm{P}_{3}$ : 905.3218; found: 905.3267 .

Synthesis of 1. A $20 \mathrm{~mL}$ scintillation vial equipped with a stir bar was charged with $\mathrm{H}_{3}{ }^{\mathrm{Ph}} \mathrm{L}(200 \mathrm{mg}, 0.221 \mathrm{mmol}$, 1 equiv) and THF $(10 \mathrm{~mL})$. A freshly prepared solution of (THF) Co[N(SiMe $\left.)_{2}\right]_{2}$ (149.7 mg, $0.332 \mathrm{mmol}, 1.5$ equiv) in THF $(10 \mathrm{~mL})$ was added dropwise with stirring. The mixture was stirred at room temperature for 12 hours, resulting in the formation of a dark green solution. All volatiles were removed under reduced pressure. The green residue was repeatedly washed with pentane, followed by diethyl ether. The remaining solid was extracted with toluene and filtered through a pad of Celite. All volatiles were removed from the filtrate under reduced pressure. The green residue was redissolved with $\mathrm{C}_{6} \mathrm{H}_{6}$, and $\mathrm{Et}_{2} \mathrm{O}$ was carefully layered on top of the $\mathrm{C}_{6} \mathrm{H}_{6}$ solution to induce crystallization. Compound $\mathbf{2}$ was isolated as large, dark green, block-shaped crystals (137.7 mg, $63 \%$ ). ${ }^{1} \mathrm{H}$ NMR $\left(\mathrm{C}_{6} \mathrm{D}_{6}, 400 \mathrm{MHz}\right): 65.76,45.15,30.57,29.54,24.78$, $24.43,24.25,21.72,21.46,17.56,14.42,13.46,12.76$, $11.59,9.25,0.29,-1.02,-6.28,-15.08,-24.34,-24.71$, $-28.07,-48.14,-63.63 \mathrm{ppm}$. Analysis calculated for $\mathrm{C}_{120} \mathrm{H}_{90} \mathrm{P}_{6} \mathrm{~N}_{6} \mathrm{Co}_{3}$ (2): C 72.86, H 4.59, N 4.25. Found: C 72.34, H 4.79, N 4.14.

Synthesis of 2. Method 1: A $20 \mathrm{~mL}$ scintillation vial equipped with a stir bar was charged with $\mathrm{H}_{3}{ }^{\mathrm{Ph}} \mathbf{L}(118 \mathrm{mg}$, $0.13 \mathrm{mmol}, 1$ equiv) and THF $(10 \mathrm{~mL})$. A freshly prepared solution of $(\mathrm{THF}) \mathrm{Co}\left[\mathrm{N}\left(\mathrm{SiMe}_{2}\right)_{2}\right]_{2}(59 \mathrm{mg}, 0.13 \mathrm{mmol}, 1$ equiv) in THF (5 mL) was added dropwise with stirring. The mixture was stirred at room temperature for 2 hours, resulting in the formation of a light green solution. A freshly prepared THF solution of $\mathrm{C}_{2} \mathrm{Cl}_{6}(32 \mathrm{mg}, 0.13 \mathrm{mmol}$, 1 equiv) that had been previously purified by sublimation was added quickly at once, resulting in the formation of a deep red solution. The mixture was stirred at room temperature for 18 hours. A small amount of an insoluble brown precipitate was removed by filtration. All volatiles were removed from the filtrate under reduced pressure. The red residue was repeatedly washed with pentane. The remaining solid was extracted with a 9:1 $\mathrm{Et}_{2} \mathrm{O}: \mathrm{C}_{6} \mathrm{H}_{6}$ mixture and filtered through a pad of Celite. All volatiles were removed from the filtrate under reduced pressure. The red residue was redissolved in $\mathrm{C}_{6} \mathrm{H}_{6}$, and pentane was carefully layered on top of the $\mathrm{C}_{6} \mathrm{H}_{6}$ solution to induce crystallization. Complex $\mathbf{2}$ was isolated as thin red plates (85 mg, $68 \%$ ). Large crystals suitable for single crystal Xray diffraction were obtained by slow vapor diffusion of $\mathrm{Et}_{2} \mathrm{O}$ into a solution of 2 in THF. ${ }^{1} \mathrm{H}$ NMR $\left(\mathrm{C}_{6} \mathrm{D}_{6}, 400 \mathrm{MHz}\right)$ : 29.4, 26.4, 24.7, 9.5, -0.1, -21.3 ppm. Analysis calculated for $\mathrm{C}_{60} \mathrm{H}_{45} \mathrm{CoN}_{3} \mathrm{P}_{3}$ : C 75.08, $\mathrm{H} 4.73, \mathrm{~N} 4.38$; found: $\mathrm{C}$ 74.61, H 4.92, N 4.20.

Method 2: A $20 \mathrm{~mL}$ scintillation vial equipped with a stir bar was charged with 1 (40 mg, $0.020 \mathrm{mmol}, 1$ equiv) and $\mathrm{C}_{6} \mathrm{H}_{6}(10 \mathrm{~mL})$. A freshly prepared solution of $\mathrm{C}_{2} \mathrm{Cl}_{6}(4.8$ $\mathrm{mg}, 0.020 \mathrm{mmol}, 1$ equiv) in $\mathrm{C}_{6} \mathrm{H}_{6}(5 \mathrm{~mL})$ was added at once with stirring. The mixture was stirred at room temperature for 3 hours, resulting in a dark red solution. All volatiles were removed under reduced pressure. The red residue was repeatedly washed with pentane, followed by diethyl ether. The remaining solid was extracted with toluene and filtered through a pad of Celite. All volatiles were removed from the filtrate under reduced pressure. The ${ }^{1} \mathrm{H}$ NMR (Figure S24) matches an authentic sample of 2 (25.4 mg, $66 \%$ ).

Method 3: A $10 \mathrm{~mL}$ Schlenk flask equipped with a stir bar was charged with 1 (40 mg, $0.020 \mathrm{mmol}, 1$ equiv) and $\mathrm{C}_{6} \mathrm{H}_{6}(10 \mathrm{~mL}$ ). The flask was degassed (freeze, pump, thaw, 3 cycles) and placed under a dry $\mathrm{O}_{2}$ atmosphere. The mixture was stirred at room temperature for 12 hours, resulting in a dark red solution. All volatiles were removed under reduced pressure. The red residue was repeatedly washed with pentane, followed by diethyl ether. The remaining solid was extracted with toluene and filtered through a pad of Celite. All volatiles were removed from 
the filtrate under reduced pressure. The ${ }^{1} \mathrm{H}$ NMR (Figure S23) matches an authentic sample of $2(11.0 \mathrm{mg}, 28 \%)$.

\section{ASSOCIATED CONTENT}

Supporting Information. Experimental procedures and characterization. This material is available free of charge via the Internet at http://pubs.acs.org.

\section{AUTHOR INFORMATION}

\section{Corresponding Author}

* rittle@berkeley.edu

\section{Notes}

The authors declare no competing financial interest.

\section{ACKNOWLEDGMENT}

This research was supported by the University of California Berkeley. We thank Dr. Nicholas Settineri for assistance with XRD, Dr. Khetpakorn Chakarawet for assistance with magnetic measurements, Prof. Kwabena Bediako for access to a SQUID magnetometer, and Eugene Kim for assistance with TGA measurements. We thank Drs. Hasan Celik, Alicia Lund, and UC Berkeley's NMR facility in the College of Chemistry (CoC-NMR) for spectroscopic assistance. Instruments in the CoC-NMR are supported in part by $\mathrm{NIH}$ S10OD024998. We thank Prof. John Anderson for helpful discussions.

\section{REFERENCES}

(1) Dehnicke, K.; Krieger, M.; Massa, W., Phosphoraneiminato complexes of transition metals. Coord. Chem. Rev. 1999, 182, 19.

(2) Dehnicke, K.; Weller, F., Phosphorane iminato complexes of main group elements. Coord. Chem. Rev. 1997, 158, 103.

(3) Berry, J. F., Terminal Nitrido and Imido Complexes of the Late Transition Metals. Comments Inorg. Chem. 2009, 30, 28.

(4) Ray, K.; Heims, F.; Pfaff, F. F., Terminal Oxo and Imido Transition-Metal Complexes of Groups 9-11. Eur. J. Inorg. Chem. 2013, 2013, 3784.

(5) Rice, N. T.; Popov, I. A.; Russo, D. R.; Bacsa, J.; Batista, E. R.; Yang, P.; Telser, J.; La Pierre, H. S., Design, Isolation, and Spectroscopic Analysis of a Tetravalent Terbium Complex. J. Am. Chem. Soc. 2019, 141, 13222.

(6) Rice, N. T.; Su, J.; Gompa, T. P.; Russo, D. R.; Telser, J.; Palatinus, L.; Bacsa, J.; Yang, P.; Batista, E. R.; La Pierre, H. S., Homoleptic Imidophosphorane Stabilization of Tetravalent Cerium. Inorg. Chem. 2019, 58, 5289.

(7) Saame, J.; Rodima, T.; Tshepelevitsh, S.; Kütt, A.; Kaljurand, I.; Haljasorg, T.; Koppel, I. A.; Leito, I., Experimental Basicities of Superbasic Phosphonium Ylides and Phosphazenes. J. Org. Chem. 2016, 81, 7349 .

(8) Stephan, D. W.; Guérin, F.; Spence, R. E. v. H.; Koch, L.; Gao, X.; Brown, S. J.; Swabey, J. W.; Wang, Q.; Xu, W.; Zoricak, P.; Harrison, D. G., Remarkably Active Non-Metallocene Ethylene Polymerization Catalysts. Organometallics 1999, 18, 2046.

(9) Stephan, D. W.; Stewart, J. C.; Guérin, F.; Spence, R. E. v. H.; $\mathrm{Xu}, \mathrm{W}$.; Harrison, D. G., Phosphinimides as a Steric Equivalent to Cyclopentadienyl: An Approach to Ethylene Polymerization Catalyst Design. Organometallics 1999, 18, 1116.

(10) Camacho-Bunquin, J.; Ferguson, M. J.; Stryker, J. M., Hydrocarbon-Soluble Nanocatalysts with No Bulk Phase: Coplanar, Two-Coordinate Arrays of the Base Metals. J. Am. Chem. Soc. 2013, 135, 5537.

(11) Engelmann, X.; Monte-Pérez, I.; Ray, K., Oxidation Reactions with Bioinspired Mononuclear Non-Heme Metal-Oxo Complexes. Angew. Chem. Int. Ed. 2016, 55, 7632.
(12) Larson, V. A.; Battistella, B.; Ray, K.; Lehnert, N.; Nam, W., Iron and manganese oxo complexes, oxo wall and beyond. Nat. Rev. Chem. 2020, 4, 404.

(13) Fang, G.-S.; Huang, J.-S.; Zhu, N.; Che, C.-M., (Nitrido)ruthenium(VI) and -osmium(VI) Complexes with Catecholate Auxiliaries: Synthesis, Structure, and Conversion into a (Phosphoraniminato)osmium(V) Complex. Eur. J. Inorg. Chem. 2004, 2004, 1341

(14) Schendzielorz, F. S.; Finger, M.; Volkmann, C.; Würtele, C.; Schneider, S., A Terminal Osmium(IV) Nitride: Ammonia Formation and Ambiphilic Reactivity. Angew. Chem. Int. Ed. 2016, $55,11417$.

(15) Wong, T.-W.; Lau, T.-C.; Wong, W.-T., Osmium(VI) Nitrido and Osmium(IV) Phosphoraniminato Complexes Containing Schiff Base Ligands. Inorg. Chem. 1999, 38, 6181.

(16) Yi; Lam, T. C. H.; Sau, Y.-K.; Zhang, Q.-F.; Williams, I. D.; Leung, W.-H., Electrophilic Ruthenium(VI) Nitrido Complex Containing Kläui's Oxygen Tripodal Ligand. Inorg. Chem. 2007, 46, 7193 .

(17) Creutz, S. E.; Peters, J. C., Spin-State Tuning at Pseudotetrahedral d6 Ions: Spin Crossover in [BP3]FeII-X Complexes. Inorg. Chem. 2016, 55, 3894.

(18) Lin, H.-J.; Siretanu, D.; Dickie, D. A.; Subedi, D.; Scepaniak, J. J.; Mitcov, D.; Clérac, R.; Smith, J. M., Steric and Electronic Control of the Spin State in Three-Fold Symmetric, FourCoordinate Iron(II) Complexes. J. Am. Chem. Soc. 2014, 136, 13326.

(19) Scepaniak, J. J.; Harris, T. D.; Vogel, C. S.; Sutter, J.; Meyer, K.; Smith, J. M., Spin Crossover in a Four-Coordinate Iron(II) Complex. J. Am. Chem. Soc. 2011, 133, 3824.

(20) Scepaniak, J. J.; Margarit, C. G.; Harvey, J. N.; Smith, J. M., Nitrogen Atom Transfer from Iron(IV) Nitrido Complexes: A Dual-Nature Transition State for Atom Transfer. Inorg. Chem. 2011, 50, 9508

(21) Bai, T.; Janes, T.; Song, D., Homoleptic iron(ii) and cobalt(ii) bis(phosphoranimide) complexes for the selective hydrofunctionalization of unsaturated molecules. Dalton Trans. 2017, 46, 12408.

(22) Alhomaidan, O.; Bai, G.; Stephan, D. W., Use of Olefin Metathesis to Link Phosphinimide-Cyclopentadienyl Ligand Complexes: Synthesis, Structure, and Ethylene Polymerization Activity. Organometallics 2008, 27, 6343.

(23) Hollink, E.; Stewart, J. C.; Wei, P.; Stephan, D. W., Ti and $\mathrm{Zr}$ bidentate bis-phosphinimide complexes. Dalton Trans. 2003, 3968.

(24) Ramos, A.; Stephan, D. W., Titanium ferrocenylphosphinimide complexes. Dalton Trans. 2010, 39, 1328.

(25) Siemeling, U.; Kölling, L.; Stammler, A.; Stammler, H.-G., Ansa-di(phosphaneiminato)titanium chelates: novel isolobal analogues of ansa-titanocene derivatives. J. Chem. Soc. Dalton Trans. 2002, 3277.

(26) Siemeling, U.; Neumann, B.; Stammler, H.-G.; Kuhnert, O., Synthesis and Structure of [ $\mathrm{NbCl} 3\{[\mathrm{NPPh} 2(\mathrm{C} 5 \mathrm{H} 4)] 2 \mathrm{Fe}\}]$ : The First Structurally Characterised Complex Containing a Chelating Di(phosphaneiminato) Ligand. Z. Anorg. Allg. Chem. 2000, 626, 825 .

(27) Hillenbrand, J.; Leutzsch, M.; Fürstner, A., Molybdenum Alkylidyne Complexes with Tripodal Silanolate Ligands: The Next Generation of Alkyne Metathesis Catalysts. Angew. Chem. Int. Ed. 2019, 58, 15690.

(28) Tsui, E. Y.; Kanady, J. S.; Day, M. W.; Agapie, T., Trinuclear first row transition metal complexes of a hexapyridyl, trialkoxy 1,3,5-triarylbenzene ligand. Chem. Commun. 2011, 47, 4189.

(29) Argent, S. P.; Tarassova, I.; Greenaway, A.; Nowell, H.; Barnett, S. A.; Warren, M. R.; Tang, C. C.; Morris, C. G.; Lewis, W.; Champness, N. R.; Schröder, M.; Blake, A. J., Assembly of high nuclearity clusters from a family of tripodal tris-carboxylate ligands. Polyhedron 2016, 120, 18. 
(30) Chao, S. T.; Lara, N. C.; Lin, S.; Day, M. W.; Agapie, T., Reversible Halide-Modulated Nickel-Nickel Bond Cleavage: Metal-Metal Bonds as Design Elements for Molecular Devices. Angew. Chem. Int. Ed. 2011, 50, 7529.

(31) Lundgren, R. J.; Peters, B. D.; Alsabeh, P. G.; Stradiotto, M., A P,N-Ligand for Palladium-Catalyzed Ammonia Arylation: Coupling of Deactivated Aryl Chlorides, Chemoselective Arylations, and Room Temperature Reactions. Angew. Chem. Int. Ed. 2010, 49, 4071.

(32) Appel, R.; Kleinstück, R.; Ziehn, K. D.; Knoll, F., Über die gemeinsame Einwirkung von Ammoniak (Aminen, Iminen) und Tetrachlorkohlenstoff auf Phosphine, I. Chem. Ber. 1970, 103, 3631.

(33) Legault, C.; Charette, A. B., Highly efficient synthesis of o(2,4-dinitrophenyl)hydroxylamine. Application to the synthesis of substituted N-benzoyliminopyridinium ylides. J. Org. Chem. 2003, 68,7119 .

(34) Mai, H.-J.; zu Köcker, R. M.; Wocadlo, S.; Massa, W.; Dehnicke, K., Phosphorane Iminato Complexes of Manganese and Cobalt with Heterocubane Structure. Angew. Chem. Int. Ed. in English 1995, 34, 1235.

(35) Anfang, S.; Harms, K.; Weller, F.; Borgmeier, O.; Lueken, H.; Schilder, H.; Dehnicke, K., Phosphaniminato-Komplexe Seltener Erden. Synthese und Kristallstrukturen von [M2(C5H5)3(NPPh3)3]-3C7H8 mit $\mathrm{M}=\mathrm{Y}$, Dy und Er. Magnetische Eigenschaften von [Dy2(C5H5)3(NPPh3)3] 3 C7H8. Z. Anorg. Allg. Chem. 1998, 624, 159.

(36) Chakarawet, K.; Atanasov, M.; Marbey, J.; Bunting, P. C.; Neese, F.; Hill, S.; Long, J. R., Strong Electronic and Magnetic Coupling in $\mathrm{M} 4(\mathrm{M}=\mathrm{Ni}, \mathrm{Cu})$ Clusters via Direct Orbital Interactions between Low-Coordinate Metal Centers. J. Am. Chem. Soc. 2020, 142, 19161.

(37) Chakarawet, K.; Bunting, P. C.; Long, J. R., Large Anisotropy Barrier in a Tetranuclear Single-Molecule Magnet Featuring Low-Coordinate Cobalt Centers. J. Am. Chem. Soc. 2018, 140, 2058.

(38) Riese, U.; Harms, K.; Pebler, J.; Dehnicke, K., Phosphaniminato-Cluster von Eisen. Die Kristallstrukturen von $[\mathrm{FeCl}(\mathrm{NPEt} 3)] 4, \quad[\mathrm{Fe}(\mathrm{C}=\mathrm{C}-\mathrm{SiMe} 3)(\mathrm{NPEt} 3)] 4 \quad$ und [Fe3Cl4\{NP(NMe2)3\}3]. Z. Anorg. Allg. Chem. 1999, 625, 746.

(39) Evans, D. F., 400. The determination of the paramagnetic susceptibility of substances in solution by nuclear magnetic resonance. J. Chem. Soc. 1959, 2003.

(40) Ellison, J. J.; Power, P. P.; Shoner, S. C., First examples of three-coordinate manganese(III) and cobalt(III): synthesis and characterization of the complexes $\mathrm{M}[\mathrm{N}(\mathrm{SiMe}) 2] 3(\mathrm{M}=\mathrm{Mn}$ or Co). J. Am. Chem. Soc. 1989, 111, 8044.

(41) Qian, D.; Hinuma, Y.; Chen, H.; Du, L.-S.; Carroll, K. J.; Ceder, G.; Grey, C. P.; Meng, Y. S., Electronic Spin Transition in Nanosize Stoichiometric Lithium Cobalt Oxide. J. Am. Chem. Soc. 2012, 134, 6096.

(42) Tsujimoto, Y.; Sathish, C. I.; Hong, K.-P.; Oka, K.; Azuma, M.; Guo, Y.; Matsushita, Y.; Yamaura, K.; Takayama-Muromachi, E., Crystal Structural, Magnetic, and Transport Properties of Layered Cobalt Oxyfluorides, $\operatorname{Sr} 2 \mathrm{CoO} 3+\mathrm{xF} 1-\mathrm{x}(0 \leq \mathrm{x} \leq 0.15)$. Inorg. Chem. 2012, 51, 4802.

(43) Chen, J.-M.; Chin, Y.-Y.; Valldor, M.; Hu, Z.; Lee, J.-M.; Haw, S.-C.; Hiraoka, N.; Ishii, H.; Pao, C.-W.; Tsuei, K.-D.; Lee, J.-F.; Lin, H.-J.; Jang, L.-Y.; Tanaka, A.; Chen, C.-T.; Tjeng, L. H., A Complete High-to-Low spin state Transition of Trivalent Cobalt

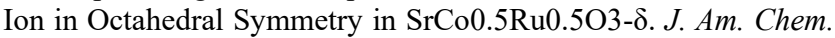
Soc. 2014, 136, 1514.

(44) Cotton, F. A.; Meyers, M. D., Magnetic and Spectral Properties of the Spin-Free 3d6 Systems Iron(II) and Cobalt(III) in Cobalt(III) Hexafluoride Ion: Probable Observation of Dynamic Jahn-Teller Effects. J. Am. Chem. Soc. 1960, 82, 5023.
(45) Boča, R., Zero-field splitting in metal complexes. Coord. Chem. Rev. 2004, 248, 757.

(46) van Leest, N. P.; Tepaske, M. A.; Oudsen, J.-P. H.; Venderbosch, B.; Rietdijk, N. R.; Siegler, M. A.; Tromp, M.; van der Vlugt, J. I.; de Bruin, B., Ligand Redox Noninnocence in [CoIII(TAML)]0/- Complexes Affects Nitrene Formation. J. Am. Chem. Soc. 2020, 142, 552.

(47) Chilton, N. F.; Anderson, R. P.; Turner, L. D.; Soncini, A.; Murray, K. S., PHI: A powerful new program for the analysis of anisotropic monomeric and exchange-coupled polynuclear d- and f-block complexes. Journal of Computational Chemistry 2013, 34, 1164.

(48) Bryan, A. M.; Long, G. J.; Grandjean, F.; Power, P. P., Synthesis, Spectroscopic Characterization, and Determination of the Solution Association Energy of the Dimer [Co $\{\mathrm{N}(\mathrm{SiMe} 3) 2\} 2] 2$ : Magnetic Studies of Low-Coordinate Co(II) Silylamides [Co $\{\mathrm{N}(\mathrm{SiMe} 3) 2\} 2 \mathrm{~L}](\mathrm{L}=\mathrm{PMe} 3$, Pyridine, and THF $)$ and Related Species That Reveal Evidence of Very Large ZeroField Splittings. Inorg. Chem. 2013, 52, 12152.

(49) Hillenbrand, J.; Leutzsch, M.; Yiannakas, E.; Gordon, C. P.; Wille, C.; Nöthling, N.; Copéret, C.; Fürstner, A., "Canopy Catalysts" for Alkyne Metathesis: Molybdenum Alkylidyne Complexes with a Tripodal Ligand Framework. J. Am. Chem. Soc. 2020, 142, 11279.

(50) Goerlich, J. R.; Schmutzler, R., Organophosphorus Compounds with Tertiary Alkyl Substituents. VI: A Convenient Method for the Preparation of di-1-Adamantylphosphine and di-1Adamantylchlorophosphine. Phosphorus Sulfur Silicon Relat. Elem. 1995, 102, 211. 


\section{Synopsis:}

A general method for the preparation of sterically encumbered, multidentate phosphinimide (PN) ligands is described. Metallation of a trigonal tris-PN platform allows for the isolation of a $\mathrm{Co}_{3}{ }^{11}$ cluster 1 . Oxidation of 1 by $\mathrm{O}_{2}$ or $\mathrm{C}_{2} \mathrm{Cl}_{6}$ cleanly furnishes a homoleptic $\mathrm{Co}^{\text {III }}$ compound 2. Compound 2 exhibits an exceedingly rare $S=2$ ground state. The role of PNs in stabilizing high spin late transition metals is investigated by XRD, magnetic susceptibility and DFT studies.

TOC:

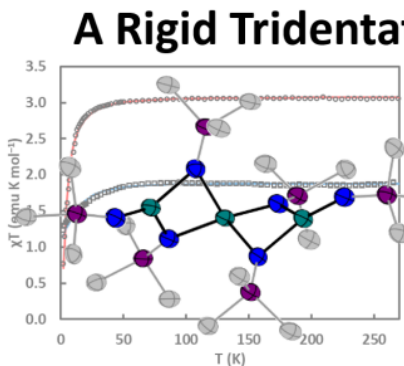

Tri-Co"l Cluster

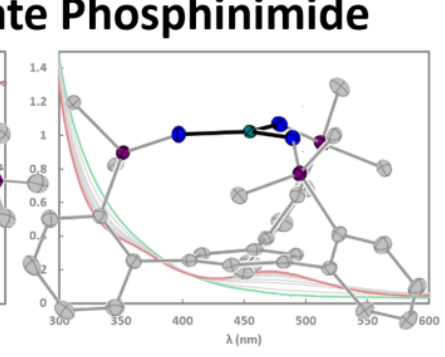

High Spin Co ${ }^{\prime \prime \prime}$ 